\title{
TORSION THEORIES AND SEMIHEREDITARY RINGS ${ }^{1}$
}

\section{DARRELL R. TURNIDGE ${ }^{2}$}

Introduction. Let $R$ be an associative ring with identity and let ${ }_{R} \mathfrak{M}$ (respectively $\mathfrak{M}_{R}$ ) denote the category of unitary left (respectively right) $R$-modules. Dickson [4] has given an axiomatic treatment of torsion for abelian categories. Specializing his definition, a torsion theory for ${ }_{R} \mathfrak{M}$ is defined to be a pair $(\mathfrak{J}, \mathcal{F})$ of classes of left $R$-modules such that

(a) $\Im \cap F=\{0\}$,

(b) $J$ is closed under homomorphic images,

(c) $\mathcal{F}$ is closed under submodules,

(d) for every left $R$-module $M$ there exists a submodule $T(M)$ of $M$ with $T(M) \in \mathfrak{J}$ and $M / T(M) \in \mathcal{F}$.

Throughout this paper two additional properties are required of a torsion theory

(e) ${ }_{R} R \in \mathcal{F}$,

(f) $J$ is closed under submodules.

A torsion theory for $\mathfrak{M}_{R}$ is similarly defined. If $(\mathfrak{J}, \mathfrak{F})$ is a torsion theory those modules in $\mathfrak{J}$ are said to be torsion and those in $\mathcal{F}$ are said to be torsion-free. Specific examples of torsion theories having these properties are the usual torsion theory for abelian groups, the torsion theory for left $R$-modules over a left Ore ring studied by Levy [9] and the $E(R)$-torsion theory considered by Jans [7].

1. Preliminaries. The addition of conditions (e) and (f) to the definition of a torsion theory makes the class $\mathfrak{I}$ a strongly complete Serre class and allows one to construct a quotient category $R \mathfrak{M} / \mathfrak{J}$ and a ring $Q_{\jmath}$ of left quotients of $R$ with respect to J. (See Gabriel [6] and the Walkers [13].) The ring of quotients of the integers $Z$ with respect to the strongly complete Serre class of torsion abelian groups is the field of rationals $Q$ and the kernel of the mapping $\eta_{G}: G \rightarrow Q$ $\otimes_{Z} G$ defined by $\eta_{G}(g)=1 \otimes g$ is the torsion subgroup $T(G)$ of $G$. In general if $(\mathcal{J}, \mathcal{F})$ is a torsion theory for ${ }_{R} \mathfrak{M}$, the kernel of the mapping

Received by the editors February 4, 1969.

1 This paper is part of the author's doctoral dissertation written under the direction of F. W. Anderson and submitted to the graduate faculty of the University of Oregon in the summer of 1968. The author wishes to express his appreciation to Professor Anderson for his encouragement and suggestions.

2 Present address: Kent State University, Department of Mathematics, Kent, Ohio 44240. 
$\eta_{M}: M \rightarrow Q_{J} \otimes_{R} M$ defined by $\eta_{M}(m)=1 \otimes m$ is contained in the torsion submodule $T(M)$ of $M$. (See [12, Proposition 1.1].) It can be shown that whenever $\operatorname{ker} \eta_{M}=T(M)$ for every left $R$-module $M$ the ring $Q_{J}$ is flat as a right $R$-module. (See [12, Theorem 1.4].) For example given any left Ore ring $R$, the ring of left quotients of $R$ with respect to the torsion theory studied by Levy is the classical ring of left quotients of $R$ which is flat as a right $R$-module. Switching our point of view, the following proposition indicates a construction of a torsion theory for ${ }_{R} \mathfrak{M}$ given any unital overring $Q$ of $R$ which is flat as a right $R$-module.

1.1. Proposition. Let $Q$ be a unital overring of $R$ which is flat as a right $R$-module. Define $\eta_{M}: M \rightarrow Q \otimes_{R} M$ by $\eta_{M}(m)=1 \otimes m$. Let $J_{l}$ $=\left\{M \in_{R} \mathfrak{M} \mid\right.$ ker $\left.\eta_{M}=M\right\}$ and $\mathscr{F}_{l}=\left\{M \in_{R} \mathfrak{M} \mid \operatorname{ker} \quad \eta_{M}=0\right\}$. Then $\left(\mathfrak{J}_{l}, \mathfrak{F}_{l}\right)$ is a torsion theory for ${ }_{R} \mathfrak{M}$.

Proof. Conditions (a), (b), (c), (e) and (f) of the definition are easily verified. For condition (d), let $M$ be a left $R$-module and let $T_{l}(M)=\operatorname{ker} \eta_{M}$. Since $Q_{R}$ is flat, we have the following commutative diagram with exact rows:

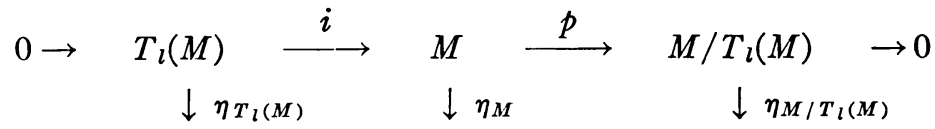

$$
\begin{aligned}
& 0 \rightarrow Q \otimes_{R} T_{l}(M) \underset{1 \otimes i}{\longrightarrow} Q \otimes_{R} M \underset{1 \otimes p}{\longrightarrow} Q \otimes_{R} M / T_{l}(M) \rightarrow 0
\end{aligned}
$$

Since $T_{l}(M)=\operatorname{ker} \eta_{M}$ we have $\eta_{M} i=(1 \otimes i) \eta_{T_{l}(M)}=0$. Thus $\eta_{T_{l}(M)}=0$ since $1 \otimes i$ is a monomorphism. Hence $T_{l}(M)=\operatorname{ker} \eta_{T_{l}(M)}$ so $T_{l}(M)$ $\in J_{l}$. Since $Q \otimes_{R} T_{l}(M)=Q \cdot \operatorname{im} \eta_{T_{l}(M)}, Q \otimes_{R} T_{l}(M)=0$. Thus $1 \otimes p$ is an isomorphism. Let $a+T_{l}(M) \in \operatorname{ker} \eta_{M / T_{l}(M)}$ with $a \in M$. Then $(1 \otimes p) \eta_{M}(a)=0$. Hence $\eta_{M}(a)=0$ so $a \in$ ker $\eta_{M}=T_{l}(M)$. Thus $a+T_{l}(M)=0$. Therefore ker $\eta_{M / T_{l}(M)}=0$ and $M / T_{l}(M) \in \mathcal{F}_{l}$.

A similar construction yields a torsion theory $\left(\mathfrak{J}_{r}, \mathfrak{F}_{r}\right)$ for $\mathfrak{M}_{R}$ given a unital overring $Q$ of $R$ which is flat as a left $R$-module.

Let $(J, F)$ be a torsion theory for $R \mathfrak{M}$ and let $M$ be a left $R$-module. Condition (d) in the definition of a torsion theory yields the exact sequence

$$
0 \rightarrow T(M) \rightarrow M \rightarrow M / T(M) \rightarrow 0
$$


of left $R$-modules with $T(M) \in J$ and $M / T(M) \in \mathcal{F}$. If $M / T(M)$ is projective this exact sequence splits and $T(M)$ is a direct summand of $M$. We are primarily interested in the following situation.

1.2. Lemma. If $(J, F)$ is a torsion theory for ${ }_{R} \mathfrak{M}$ such that every finitely generated torsion-free left $R$-module is projective, then $T(M)$ is a direct summand of $M$ for every finitely generated left $R$-module $M$.

2. Applications to semihereditary rings. A ring $R$ is said to be left (respectively right) semihereditary if every finitely generated left (respectively right) ideal of $R$ is projective. Any ring $R$ having a torsion theory satisfying the hypothesis of (1.2) is left semihereditary since the left ideals of $R$ are torsion-free as left $R$-modules. By [ 1 , Proposition VI, 2.9] if $R$ is a left (or right) semihereditary ring, then GWD $R \leqq 1$.

2.1. Theorem. Let $(\mathfrak{J}, \mathfrak{F})$ be a torsion theory for ${ }_{R} \mathfrak{M}$. Suppose that $Q_{\jmath}$ is von Neumann regular and flat as a left $R$-module. Then the following are equivalent.

(i) $R$ is right semihereditary,

(ii) GWD $R \leqq 1$,

(iii) ${ }_{R} M$ is flat for all $M \in \mathfrak{F}$.

Proof. (i) implies (ii). This follows from [1, Proposition VI, 2.9].

(ii) implies (iii). Let ${ }_{R} M \in \mathcal{F}$ and let $I$ be any right ideal of $R$. Since $Q_{J}$ is flat as a left $R$-module

$$
0 \rightarrow I \otimes_{R} Q \jmath \rightarrow R \otimes_{R} Q \jmath
$$

is an exact sequence of right $Q_{J}$-modules. Moreover, $Q_{J} \otimes_{R} M$ is flat as a left $Q_{\mathfrak{J}}$-module since $Q_{\mathfrak{J}}$ is von Neumann regular, so the sequence

$$
0 \rightarrow\left(I \otimes_{R} Q_{\jmath}\right) \otimes_{Q_{T}}\left(Q_{\jmath} \otimes_{R} M\right) \rightarrow\left(R \otimes_{R} Q_{\jmath}\right) \otimes_{Q_{T}}\left(Q_{\jmath} \otimes_{R} M\right)
$$

is exact. By associativity of the tensor product

$$
0 \rightarrow I \otimes_{R}\left(Q \jmath \otimes_{R} M\right) \rightarrow R \otimes_{R}\left(Q_{\jmath} \otimes_{R} M\right)
$$

is exact. Hence $Q_{J} \otimes_{R} M$ is flat as a left $R$-module. Define $\eta_{M}: M$ $\rightarrow Q_{J} \otimes_{R} M$ by $\eta_{M}(m)=1 \otimes m$. Since $T(M)=0$ and ker $\eta_{M} \subseteq T(M), \eta_{M}$ is a monomorphism. Letting $K=\left(Q_{J} \otimes_{R} M\right) / \eta_{M}(M)$ we have the following exact sequence of left $R$-modules

$$
0 \rightarrow M \rightarrow Q^{\jmath} \otimes_{R} M \rightarrow K \rightarrow 0 .
$$

Then for each right $R$-module $X$, the sequence 


$$
\operatorname{Tor}_{2}^{R}(X, K) \rightarrow \operatorname{Tor}_{1}^{R}(X, M) \rightarrow \operatorname{Tor}_{1}^{R}\left(X, Q_{\jmath} \otimes_{R} M\right)
$$

is exact. By the preceding $\operatorname{Tor}_{1}^{R}\left(X, Q_{j} \otimes_{R} M\right)=0$. Moreover $\operatorname{Tor}_{2}^{R}(X, K)$ $=0$ since GWD $R \leqq 1$. Hence $\operatorname{Tor}_{1}^{R}(X, M)=0$ for every right $R$ module $X$, so ${ }_{R} M$ is flat for each $M \in \mathcal{F}$.

(iii) implies (i). By [4, Theorem 2.3], $\mathcal{F}$ is closed under direct products. Thus every submodule of a direct product of copies of ${ }_{R} R$ is in F. Thus every torsionless left $R$-module is flat and by [2, Theorem 4.1] $R$ is right semihereditary.

2.2. Theorem. Let $(\mathfrak{J}, \mathfrak{F})$ be a torsion theory for ${ }_{R} \mathfrak{M}$. Suppose that $Q_{J}$ is semisimple and flat as a left $R$-module. Then the following are equivalent.

(i) $R$ is right semihereditary,

(ii) $R$ is left semihereditary,

(iii) GWD $R \leqq 1$,

(iv) ${ }_{R} M$ is flat for all $M \in \mathcal{F}$,

(v) ${ }_{R} M$ is projective for all finitely generated $M \in \mathcal{F}$.

Proof. The equivalence of (i), (iii) and (iv) follows from (2.1). (ii) implies (iii). This follows from [1, Proposition VI, 2.9]. (v) implies (ii). This is immediate since every left ideal of $R$ is in $\mathfrak{F}$.

(iv) implies (v). Since $Q_{J}$ is semisimple, it is isomorphic to the maximal ring of left quotients of $R$ and we have $Z\left({ }_{R} R\right)=0$. (See [10].) Let ${ }_{R} M \in \mathfrak{F}$ be finitely generated. By (iv) ${ }_{R} M$ is flat. Since $Q_{J}$ is semisimple, $Q_{J} \otimes_{R} M$ is a projective left $Q_{J}$-module. Thus by [11, Theorem 2.8] ${ }_{R} M$ is projective.

2.3. Corollary. Let $(J, \mathcal{F})$ be as in (2.2). If any of the equivalent conditions of (2.2) are satisfied, then $T(M)$ is a direct summand of $M$ for every finitely generated left $R$-module $M$.

Proof. See (1.2).

In the following $\left(J_{l}, F_{l}\right)$ and $\left(\mathfrak{J}_{r}, \mathcal{F}_{r}\right)$ denote torsion theories for ${ }_{R} \mathfrak{M}$ and $\mathfrak{M}_{R}$ respectively constructed as in (1.1).

2.4. Theorem. Suppose $R$ is a unital subring of a von Neumann regular ring $Q$ which is left and right $R$-flat. Then the following are equivalent.

(i) $R$ is right semihereditary,

(ii) $R$ is left semihereditary,

(iii) GWD $R \leqq 1$,

(iv) $\mathscr{F}_{l}=\left\{\left.M \in_{R} \mathfrak{M}\right|_{R} M\right.$ is flat $\}$,

(v) $\mathcal{F}_{r}=\left\{N \in \mathfrak{M}_{R} \mid N_{R}\right.$ is flat $\}$. 
Proof. (i) implies (iii) and (ii) implies (iii). These follow from [1, Proposition VI, 2.9].

(iii) implies (iv). Suppose ${ }_{R} M$ is flat. Then the mapping $\eta_{M}: M$ $\rightarrow Q \otimes_{R} M$ is a monomorphism, so $M \in \mathcal{F}_{l}$. The proof of (ii) implies (iii) in (2.1) is easily adapted to show that ${ }_{R} M$ is flat for each $M \in \mathcal{F}_{l}$.

(iii) implies (v). This is proved using an argument similar to (iii) implies (iv).

(iv) implies (i) and (v) implies (ii). These are proved using arguments similar to (iii) implies (i) in (2.1).

Sandomierski [11, Theorem 2.10] has shown that a ring $R$ is left semihereditary if and only if GWD $R \leqq 1$ and the maximal ring of left quotients of $R$ is von Neumann regular and right $R$-flat. Using this and taking $Q$ to be the maximal ring of left quotients in (2.4) we have the following.

2.5. Corollary. If $R$ is left semihereditary and the maximal ring of left quotients of $R$ is left $R$-flat, then $R$ is right semihereditary.

Suppose that $R$ has a two-sided maximal ring of quotients $Q$ which is semisimple. Then by [10, Theorem 2.7] $Q$ is flat as both a left and as a right $R$-module. Moreover, constructing $\left(\mathfrak{J}_{l}, \mathfrak{F}_{l}\right)$ and $\left(\mathfrak{J}_{r}, \mathfrak{F}_{r}\right)$ as in (1.1), $T_{l}(M)=Z\left(_{R} M\right)$ for every left $R$-module $M$ and $T_{r}(N)=Z\left(N_{R}\right)$ for every right $R$-module $N$ by [10, Theorem 2.3] where $Z(A)$ is the singular submodule of a module $A$. (See [10].)

2.6. Theorem. Suppose that $R$ has a two-sided maximal ring of quotients $Q$ which is semisimple. Then to the list of equivalences of (2.4) can be added

(vi) ${ }_{R} M$ is projective for all finitely generated $M \in \mathcal{F}_{l}$,

(vii) $N_{R}$ is projective for all finitely generated $N \in \mathcal{F}_{r}$.

Proof. See the proof of (2.2).

2.7. Corollary. If $R$ is a left (equivalently right) semihereditary ring having a semisimple two-sided maximal ring of quotients, then $Z\left(_{R} M\right)$ is a direct summand of $M$ for every finitely generated left $R$ module $M$ and $Z\left(N_{R}\right)$ is a direct summand of $N$ for every finitely generated right $R$-module $N$.

Levy [9, Theorem 6.1] obtained the preceding result for rings having a semisimple two-sided classical ring of quotients.

3. Examples. A semihereditary commutative integral domain is called a Prüfer ring. Since every commutative integral domain has a 
(semisimple) field of quotients, the Prüfer rings can be characterized as those commutative integral domains for which every finitely generated torsion-free (in the classical sense) module is projective. Hence the (classical) torsion submodule of every finitely generated module over a Prüfer ring is a direct summand. Kaplansky [6] has shown that this property characterizes the Prüfer rings among the commutative integral domains.

Endo [5] has shown that a commutative ring $R$ is semihereditary and has a semisimple classical ring of quotients if and only if it is a finite direct sum of Prüfer rings. For $n>1$ the ring $M_{n}(R)$ of $n \times n$ matrices over such a ring $R$ gives an example of a noncommutative semihereditary ring having a two-sided semisimple classical ring of quotients.

Colby and Rutter [3, Theorem 3.2] have shown that every left hereditary ring $R$ for which the injective envelope of ${ }_{R} R$ is projective is isomorphic to a finite direct sum of complete blocked triangular matrix rings over division rings. Any such ring is semihereditary and has a semisimple two-sided maximal ring of quotients.

If $D$ is any PID with field of quotients $Q$ the ring of all $2 \times 2$ matrices

$$
\left(\begin{array}{ll}
d_{1} & q \\
0 & d_{2}
\end{array}\right)
$$

with $d_{1}, d_{2} \in D$ and $q \in Q$ is semihereditary and has the ring of $2 \times 2$ upper triangular matrices over $Q$ as a two-sided classical ring of quotients. This ring has $M_{2}(Q)$ as a semisimple two-sided maximal ring of quotients.

\section{REFERENCES}

1. H. Cartan and S. Eilenberg, Homological algebra, Princeton Univ. Press, Princeton, N. J., 1956. MR 17, 1040.

2. S. U. Chase, Direct products of modules, Trans. Amer. Math. Soc. 97 (1960), 457-473. MR 22 \#11017.

3. R. R. Colby and E. A. Rutter Jr., Generalizations of QF-3 rings (to appear).

4. S. E. Dickson, A torsion theory for Abelian categories, Trans. Amer. Math. Soc. 121 (1966), 223-235. MR 33 \#162.

5. S. Endo, On semi-hereditary rings, J. Math. Soc. Japan 13 (1961), 109-119. MR 25 \#2107.

6. P. Gabriel, Des catégories abéliennes, Bull. Soc. Math. France, 90 (1962), 323448.

7. J. P. Jans, Some aspects of torsion, Pacific J. Math. 15 (1965), 1249-1259. MR 33 \#163.

8. I. Kaplansky, A characterization of Prïfer rings, J. Indian Math. Soc. 24 (1960), 279-281. MR 23 \#A2443. 
9. L. Levy, Torsion-free and divisible modules over non-integral-domains, Canad. J. Math. 15 (1963), 132-151. MR $26 \# 155$.

10. F. L. Sandomierski, Semisimple maximal quotient rings, Trans. Amer. Math. Soc. 128 (1967), 112-120. MR 35 \#5473.

11. - Nonsingular rings, Proc. Amer. Math. Soc. 19 (1968), 225-230. MR 36 \#2648.

12. D. R. Turnidge, Torsion theories and rings of quotients of Morita equivalent rings (to appear).

13. E. A. Walker and C. Walker, Quotient categories and rings of quotients (to appear).

\section{UNIVERSITY OF OREGON}

